\title{
The use of computer-based training tools in Europe - an overview and new approaches
}

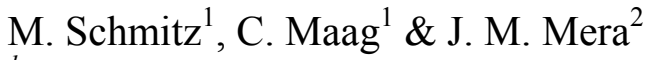 \\ ${ }^{1}$ Center for Traffic Sciences, University of Wuerzburg, Germany \\ ${ }^{2}$ CITEF, Polytechnic University of Madrid, Spain
}

\begin{abstract}
In recent years, the use of driving simulation has become one of the most effective tools for the training of train drivers. The European project 2TRAIN aims at creating software modules to enhance the training and assessment capabilities of driving simulators. As these software modules should be highly compatible with existing simulator systems, developers need to gather specific information about technical features that are most relevant for the application of simulator-based railway training. To reach this aim railway companies have been interviewed in order to provide detailed information connected with technical, organisational and content-related characteristics of applied training. One of the main findings is that the technical features and capabilities of simulators are extremely diverse as each company is focused on different training objectives. As a consequence each operator demands different technical requirements. This paper presents the users' view on the technological solutions, the fields of application, a comparison with other training methods and comments on shortcomings and future improvements. Recommendations are extracted in order to improve computer-based training in an effective and affordable way.
\end{abstract}

Keywords: computer-based training, driving simulation, rail human factor.

\section{Introduction}

The establishment of safe, competitive, and interoperable railways in Europe requires particular attention to the qualification of train drivers. In order to strengthen a European harmonisation in rail traffic it is appropriate to advance (1) common training technology as well as (2) common training concepts. Developments concerning these two aspects are the scientific and technological 
objectives of 2TRAIN. The project is funded by the European Commission under FP6 [1].

Individual European countries differ in national laws, engine technology, signalling systems, rule books, and general training structures. As a consequence of this diversity, a complete harmonisation of training technology and training contents will be unachievable. Nevertheless, as Europe grows together and crossborder operations increase there is a strong need to harmonise and coordinate the education and training of train drivers concerning general driving and operational abilities as well as particular crisis management competencies. Taking into account experiences made in the past, 2TRAIN aims at developing European best-practice solutions for an efficient, safety enhancing and cost-effective use of modern technologies for training as well as for the ongoing competence and performance assessment [2].

Important benefits can be obtained by the use of computer-based systems and simulators in training [3]. These computer-based technologies facilitate the establishment of common training efforts for train drivers in Europe and enable an enhancement of training efficiency by using interactive training of realistic situations [4]. In contrast to training in real environment, the training session can variably be composed of different technical failures and hazard operational situations of which many cannot be trained in reality. Furthermore, it is possible to replicate scenarios at any given time. Several safety-related and economic advantages of simulation contribute to the wide distribution of this training method. In the near future, railway companies will even intensify the usage of simulators for the training and assessment of train drivers.

A fundamental objective of 2TRAIN is the benchmarking of (1) training tools and technologies as well as (2) training concepts already in use in different European countries. The state of the art concerning training technology (e.g. simulator hardware, architecture, and software) and drivers' competencies and training contents is analysed by reviewing the relevant publications. Subsequently, data about training systems of European railway undertakings is collected with a special focus on simulation and training contents related to driving under abnormal/irregular conditions as well as human factor contents. By these two steps, the current status and quality of train driver training is evaluated and the requirements for future computer-based training concepts are specified.

The benchmarking process aims at describing the present situation of computerbased training in different companies and the settings under which the training is applied. The benchmarking methodology and selected key findings are presented and discussed in the following sections. The detailed results are summarised in a benchmarking report on computer-based railway training in Europe [5].

\section{Benchmarking process}

\subsection{Method}

The benchmarking process consists of several phases of research. Figure 1 illustrates the structure of the benchmarking process in the frame of 2TRAIN. 
The data gathering itself is a central part of the benchmarking and follows a twostep approach.

The first step consists of a screening questionnaire sent to 75 companies in more than 20 European countries. This screening questionnaire aims at giving a preliminary look at the use of simulation and other e-learning tools across Europe.

Based on the results of this first questionnaire, a sample of 18 railway companies was selected for a more detailed face-to-face interview. The interviews took place from April to June 2007. A detailed key question form (KQF) was prepared mainly with the objective of obtaining the most comprehensive overview of (1) training contents for train drivers, (2) tools used for training, and (3) organisation of training. As different interviewers with different professional background conducted the benchmarking interviews the objective of the KQF was to guarantee a standardised interview structure and a common analysis of the gathered data.

One part of the KQF is compiled out of multiple choice questions asking for the training methods used for specific training topics. Another part of the questionnaire requires the respondent to estimate the amount of current training on several topics by means of different training methods.

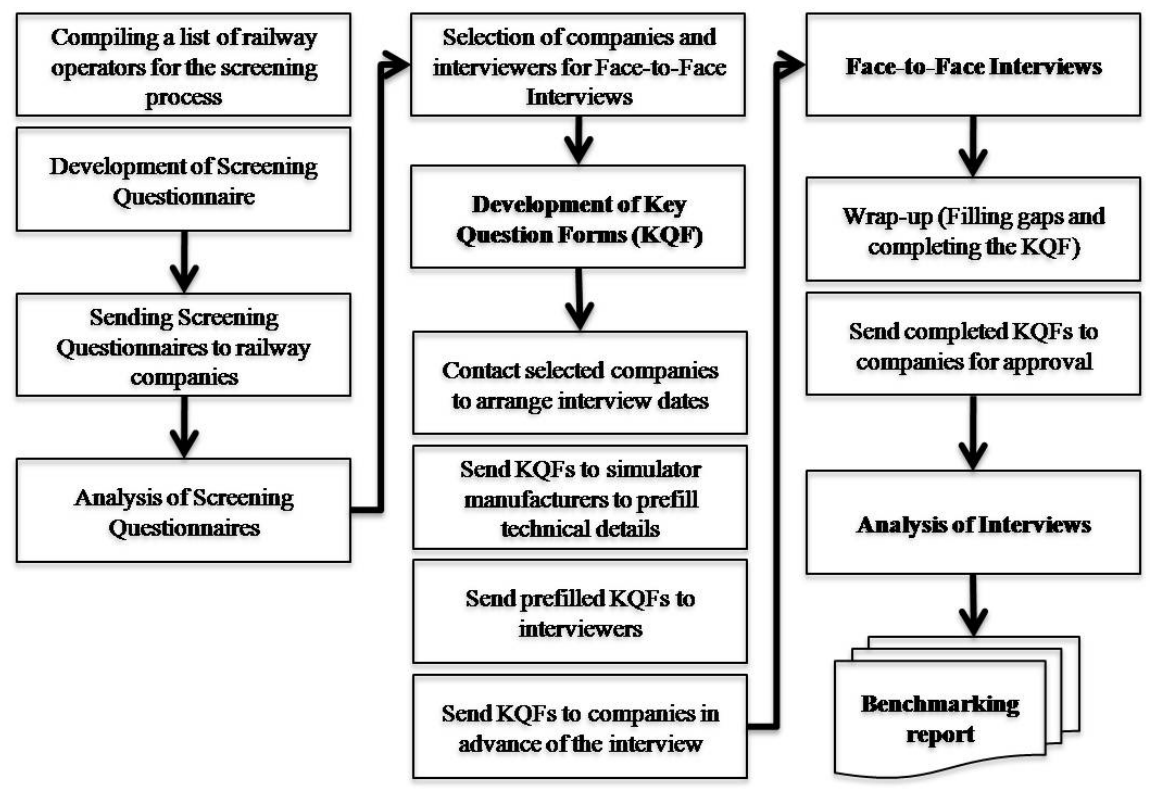

Figure 1: $\quad$ Structure of the benchmarking process.

Furthermore, the respondent has to give information about the amount of simulator usage in the course of initial training, advanced training, and competence check. Training modules dealing with abnormal and irregular conditions of railway operation, emergency situations, and human factors 
training should be described in detail by using a template asking for learning objectives, the description of the training content, target group, duration, training method, trainer-trainee ratio, and for the assessment procedure. The last part of the KQF covers general and organisational aspects of the training concept, e.g. development of training, regulations, assessment scheme, evaluation of training, and encountered problems.

All questionnaires were wrapped-up in order to fill gaps and to complete the KQFs. The completed forms were sent to the interviewed companies for approval before the main analysis of the benchmarking results took place. Finally, the whole process ended in the compilation of the final benchmarking report [5].

\subsection{Sample}

18 companies (České dráhy, CFL, Deutsche Bahn, Irishrail, MÁV, Metro de Barcelona, Metro de Madrid, Norges Statsbaner, Renfe, S-Bahn Berlin, Scotrail, SNCF, SNCB, Southern, SouthWestTrains, Stuttgarter Straßenbahnen, Trenitalia, VAG Nuremberg) were selected for the detailed face-to-face interviews. Most of the interviewed companies have many years of experience in running training simulation and other e-learning tools. The sample represents the European railway area as the selected companies employ more than 600000 people. This number includes more than 77000 train drivers. Not only major railway undertakings but also metro and tram operators of different European countries were part of the research sample. The study is based on the analysis of 138 simulator systems developed by eight different manufacturers: Citef, Corys T.E.S.S., EADS, Indra, Krauss-Maffei Wegmann, Lander, OKTAL, and Sydac.

\section{Results}

In the following section, the results of the benchmarking interviews are presented. The first part deals with results related to the training technology. A topic of special interest was to display the expectations and experiences of the interviewed companies. The second part describes the main fields of simulator usage and compares computer-based training with conventional training methods. The last section is focused on shortcomings of the existing technology, on feedback about the day-to-day use of simulation technology, and on the intention of future improvements.

\subsection{Training simulation technology - the users' view}

Five different types of simulators are differentiated in the frame of the study: (A) software interface of cab equipment, (B) part-task trainer, (C) partial cab, (D) full replica cab without motion system, and (E) full replica cab with motion system. About $35 \%$ of the analysed systems are partial cab simulators, another $35 \%$ simulators are equipped with a full replica cab and motion system, and $17 \%$ are equipped with a full replica cab but without a motion system. Software interfaces are used in $9 \%$ and part-task trainers due to the limited field of 
application in only $4 \%$ of the sample. Several companies have decided for a combination of simulators in terms of scalability as to cover the wide range of applications up to a full-mission simulator on the one hand, and to guarantee a high amount of simulator training by cost-effective partial-cab solutions.

The eighteen companies were asked to rate the importance of some technical features to be expected from a training simulator on a five point scale $(1=$ "not important", 5 = "very important"). The most important features with average ratings above 4.5 are the realistic train behaviour and the possibility of changing settings during an exercise. Average ratings above 4.0 are given for an objective assessment system, real cabin controls, realistic tracks and signalisation, realistic visualisation and sound, different driving and weather conditions. Average ratings above 3.0 are stated for the existence of a full replica cab, for the modelling of all subsystems (e.g. electrical, pneumatic), for the possibility of simulating different rolling stock, and for compliance with compatibility standards. The lowest average ratings below 3.0 are given for the existence of a motion system, the ability to connect different simulators for combined exercises, the existence of real surroundings as well as the representation of road traffic, passengers and pedestrians. It has to be considered that the scoring on some of these items is greatly dispersed. For example, the motion system is of high importance for those operators running a simulator with motion system but is nearly unnecessary to other companies.

During the interviews, the operators were also asked to evaluate the existing training technology on a six point scale $(0=$ "simulator does not have the characteristic", 1 = "Not realistic", 5 = "very close to reality") with regard to how close the existing simulators are to reality. The representation of road traffic, pedestrians and passengers are the lower valuated items due to the difficulty of emulating human behaviour on the one hand, and the low importance that most operators place on these topics on the other. The mathematical train model, the signalisation system and the similarity between simulator and real train cabin (including movement system) are the best rated items. The model of other trains apart from the ego train, the ability to simulate failures, and the visual/sound system only received medium-high marks.

Finally, the day-to-day usage of the simulators should be rated $(0=$ "not available", 5 = "maximal customer satisfaction"). It could be found that the operators give good marks for the improvement of training-learning capacity due to simulators and for the amount of information given to the instructor during the exercise. The less valued items are the quality of the final exercise report and the existing facilities to create new exercises. A recording system, the time needed to load the simulator exercise and the general manageability and ease of use receives medium marks.

\subsection{Use of training simulators}

In average, about $40 \%$ of all simulator sessions are allocated for initial training. Therefore, initial training is the main topic trained by means of a simulator. Besides that, simulator training is an instrument for advanced training $(\sim 30 \%)$ 
and competence check ( $24 \%)$. The remaining $6 \%$ can be allocated to topics like acquisition of route knowledge, training on rolling stock or ATC/ATP systems.

The use of simulator training in comparison to other training methods such as classroom or real cab in concrete phases and for specific topics of training is rather inconsistent. As a training method for initial training, simulators are only used in $7 \%$ of the overall training amount. Concerning advanced training, the companies cover in average $25 \%$ of the complete training with simulation. $26 \%$ of all competence checks are realised by means of a simulator (see Figure 2). These findings underline the general advantages of simulator-based training, i.e. the realisation of emergency situations and driving under abnormal and degraded conditions.

In all companies, conventional training methods - as classroom or training on the real cab - still play an important role, especially for those areas that cannot be fully substituted by a simulator due to national or companies' directives, e.g. route knowledge, rolling stock training (see Figure 3).

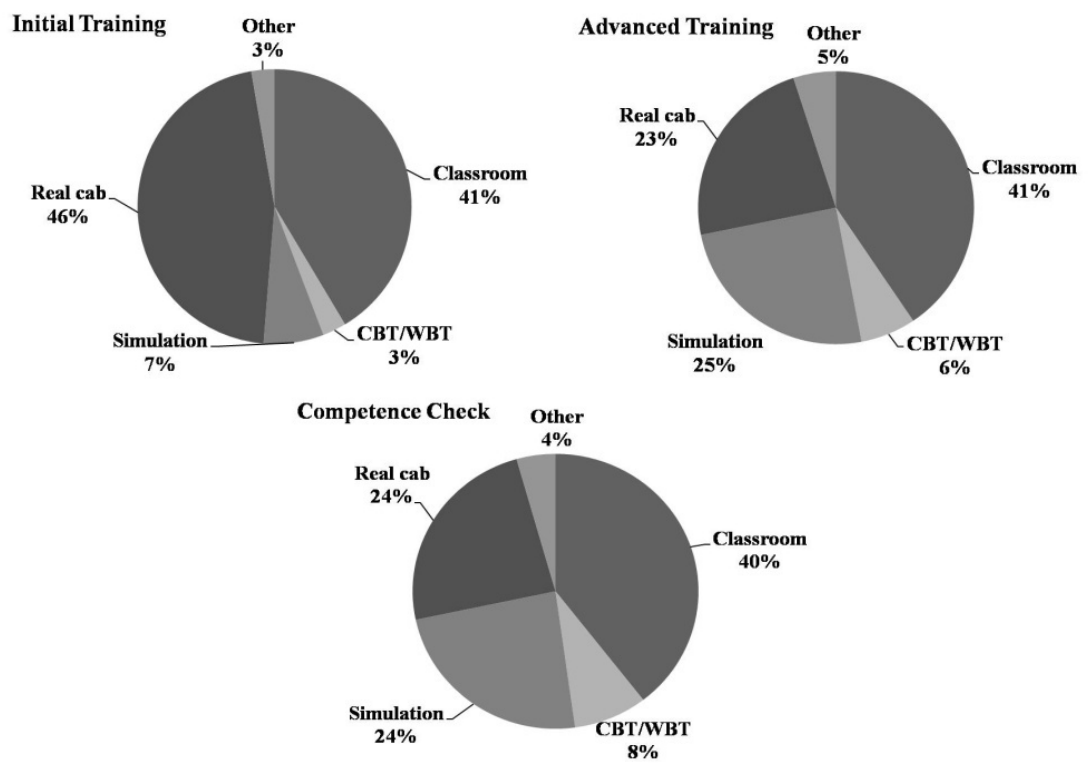

Figure 2: $\quad$ Comparison of different training methods and training contents.

The average number of hours a train driver spends on a simulator within the scope of initial training is approximately 30 hours with some companies considerably above average (up to 160 hours per trainee). Simulators are used for advanced training only to a limited extent in terms of hours per year (one to eight hours per trainee per year) due to a high number of drivers in many railway undertakings and the limited resources of training hours in the simulator. Another reason is that advanced training in the simulator is not obligatory in all companies. Nevertheless, in average nearly every fourth hour of advanced training is realised as simulator exercise. Simulators are also used for 

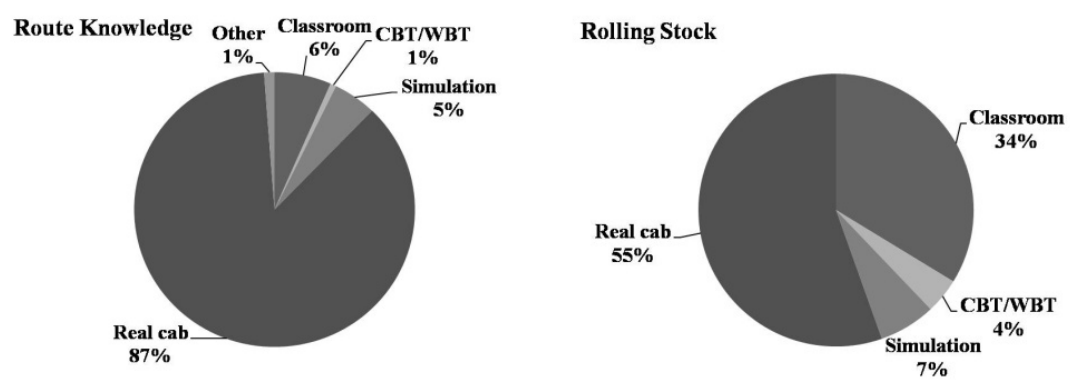

Figure 3: Use of simulation for route knowledge and rolling stock training.

competence check, i.e. for a regular performance assessment of the train driver. 10 out of 17 companies use the simulator for this training topic with a range of half an hour to three hours per trainee per year.

\subsection{Organisational issues, shortcomings and future planning}

The last part of the interviews deals with organisational issues, evaluation approaches, and users' comments concerning shortcomings of the existing training technology as well as future improvements.

Companies were asked for any evaluation studies conducted in the past. Such studies should clarify if the training is interesting and motivating for the trainee and if the training achieves the expected results in terms of knowledge transfer, behavioural change, skills and competence development. Besides the fact that some companies have introduced a regular feedback instrument (paper-pencil) asking for the trainees' acceptance of the computer-based training sessions, no company evaluates the training effects in a systemic and standardised way.

Each company states certain insufficiencies regarding the operation, maintenance and development of simulator training. The answers can be divided into (1) technical and economical shortcomings, (2) shortcomings related to the assessment capabilities, (3) feedback given by the trainees, (4) feedback given by the labour unions, and (5) the intention of future improvements.

The given answers imply that it is difficult to maintain the simulator at the level of the real system (according to changes affecting the infrastructure or the rolling stock) as implementing changes in the simulation is often cost expensive and time consuming and can only be done by the manufacturer. The realisation of a generic driver's cab and a generic infrastructure is suggested as a reasonable solution to achieve a higher flexibility in terms of developing new exercises.

Other comments respond to the quality of post-run analyses as there is not enough feedback and assessment data for the instructor. A simpler scoring report with an option of focusing on errors could be one solution. At some training simulators an automated objective assessment system is totally missing. As a consequence, the use of objective performance markers for the assessment procedure and a connection of the assessment database with the company's information system are proposed. 
Concerning the trainees' feedback, some drivers ask for more sessions on the simulator and demand for an immediate re-training after an incorrect action. The realism of simulation should be as high as possible. Due to a missing full motion system, some existing drivers are not keen on simulators as the sensation of braking is limited.

The feedback given by labour unions and staff councils is inconsistent. At some companies an agreement with the staff council has been settled not to use the simulator for the surveillance of train drivers. At other companies, unions do not allow the detailed performance check by using objective assessment data. Furthermore, simulator sessions can only be attributed as additional training and should never reduce the amount of conventional methods. Other companies quote that the acceptance and compliance are quite high. Good experience was made with the involvement of the labour unions from the beginning on as part of the simulator steering group.

Asking for future improvements and planning some companies argue for an intensified use of simulation in the course of initial training, for training sessions focused on the distraction of drivers in critical phases, and for implementing cross-border training on partial cab simulators. Other companies would like to increase the number of simulated engine types and realise a higher number of small desk simulators aiming at a whole fleet of simulators.

\section{Summary and conclusions}

The objective of 2TRAIN is to give best-practice guidelines for an efficient, safety enhancing and cost-effective use of modern technologies for the training of train drivers as well as for the ongoing competence and performance assessment. The objective of the benchmarking process is to provide a comprehensive overview of the training concepts used in different railway companies in different European countries. At the same time the objective is to gain some understanding of the training background in the individual interviewed companies.

The benchmarking results are based on a structured research process and interviews with 18 European railway undertakings (including light rail and metro companies). The technical features and capabilities of simulators are extremely diverse as each company is focused on different training objectives. Some companies combine full-mission simulators with cost-effective partial-cab solutions whereas others are only focussed on a single type. All in all, the analysis highlights the important role of simulation for the training and assessment of train drivers. Nevertheless, the interviewed companies state some shortcomings that hinder an optimal use of the existing training technology. Major points of criticism are the missing possibility to create new exercises and scenarios as well as the assessment capabilities of the simulators, in particular the quality of the simulator reports.

As a recommendation, an optimal benefit could be achieved if the simulator training is embedded into the overall competence management of the company. In addition, the simulator exercises should be tailored to the intended learning 
objectives. Furthermore, it is important that the train drivers become familiar with the new technology by using the training tools regularly in the course of initial training, advanced training, and competence check. Standardised scenarios and procedures for all training centres of a company lead to a transparent training and assessment. The use of simulator data supports an objective and detailed assessment of the train drivers' performance.

Simulator training is an effective method within a blended learning environment that has the major objective to guarantee highly qualified railway personnel that is able to handle all kinds of operational situations in a safe and appropriate way.

\section{References}

[1] 2TRAIN, www.2train.eu

[2] Schmitz, M. \& Maag, C., 2TRAIN - TRAINing of TRAIN drivers in safety relevant issues with validated and integrated computer-based technology. Proc. of the 15 $5^{\text {th }}$ International Symposium EURNEX - Žel 2007 (Bd. 2), EDIS: Žilina, pp. 206-211, 2007.

[3] Rail Safety \& Standards Board (2007). Good practise on simulation as a tool for training and assessment. Railway Group Standard RS/501, Issue 2, June 2007.

[4] European Commission (2007b). Directive 2007/59/EC of the European Parliament and of the Council of 23 October 2007 on the certification of train drivers operating locomotives and trains on the railway system in the Community. Luxembourg: Official Journal of the European Union.

[5] 2TRAIN Consortium (2008). Benchmarking report on computer-based railway training in Europe. Project report: www.2train.eu. 